\title{
Finite order approximations to radiation forces for wave energy applications
}

\author{
A. Roessling \& J.V. Ringwood \\ Centre for Ocean Energy Research, Maynooth University, Maynooth, Ireland
}

\begin{abstract}
This paper reviews different possibilities to obtain the radiation forces for time-domain simulations of wave energy converters or other floating structures. Three different ways to calculate the radiation forces using boundary element methods are compared in the time- and frequency-domain. The three boundary element programs are also compared with respect to their computational cost. To approximate the radiation forces, two different methods are investigated, Prony's method in the time-domain, and frequency domain identification in the frequency-domain.
\end{abstract}

\section{INTRODUCTION}

For wave energy applications, hydrodynamic boundary element methods (BEMs) are used for a variety of purposes. Applications include frequencydomain representations of wave energy systems and time-domain simulations. Examples for frequencydomain applications are the use of response amplitude operators and power calculations. An intermediate step often employed, is a finite-order approximation of the nonparametric hydrodynamic parameters calculated by the BEMs. Boundary element software packages can solve the hydrodynamic problem either in the time-domain, as for example Achil3D, or in the frequency-domain, as for example Wamit and Nemoh. However, in general, the use of parametric approximation methods, and the original BEM domain, are agnostic in relation to the final application domain and requirements. For example, a frequencydomain BEM is often employed, in conjunction with a frequency-domain approximation method to produce a model for time-domain simulations. This paper takes an overview of the radiation force modeling landscape, which is depicted in figure 1 , and makes a critical examination of the routes through this landscape, with a focus on application requirements in time-domain simulations.

In general, time-domain simulations of wave energy converters and other floating structures, are based on the Cummins equation. This is a differential equation were the radiation term is represented by a convolution integral, accounting for fluid-memory effects. The direct calculation of the convolution integral in a time-domain simulation is computationally expensive and inconvenient (Taghipour et al. 2008).
Therefore, the radiation forces are usually approximated in time-domain simulations.

The three basic steps that can be applied to use radiation forces in time-domain simulations are summarized in figure 1. The first step is to calculate the radiation forces using hydrodynamic software. In this paper Achil3D, Wamit and Nemoh are used for this purpose. In the second step, the radiation forces are approximated. Here Prony's method and frequency domain identification are used. The third step is the actual use of the radiation forces in time-domain simulations. As shown in figure 1, steps one and two can be carried out either in the time- or frequency-domain. Time- and frequency-domain are related by Fourier transformations, often referred to as Ogilvie's relations (see equations (3) to (5)).

In its general form the time-domain equation of motion for a floating body, with zero forward speed, is described by the Cummins equation (Cummins 1962):

$$
\boldsymbol{f}_{e x c}(t)=\left(\boldsymbol{M}+\boldsymbol{A}_{\infty}\right) \ddot{\boldsymbol{x}}(t)+\int_{0}^{t} \boldsymbol{K}(t-\tau) \dot{\boldsymbol{x}}(\tau) d \tau+\boldsymbol{C} \boldsymbol{x}(t)
$$

Where $\boldsymbol{f}_{\text {exc }}$ is the excitation force due to the incoming waves, $M$ is the mass matrix of the rigid floating body, $\boldsymbol{A}_{\infty}$ is the added mass at infinite radian frequency $\omega$, and $C$ is the hydrostatic stiffness matrix. The vectors $\boldsymbol{x}, \dot{\boldsymbol{x}}$ and $\ddot{\boldsymbol{x}}$ denote the position, velocity and acceleration of the rigid body, respectively. The kernel $\boldsymbol{K}(t-\tau)$ of the convolution integral is the matrix of impulse-response functions. 


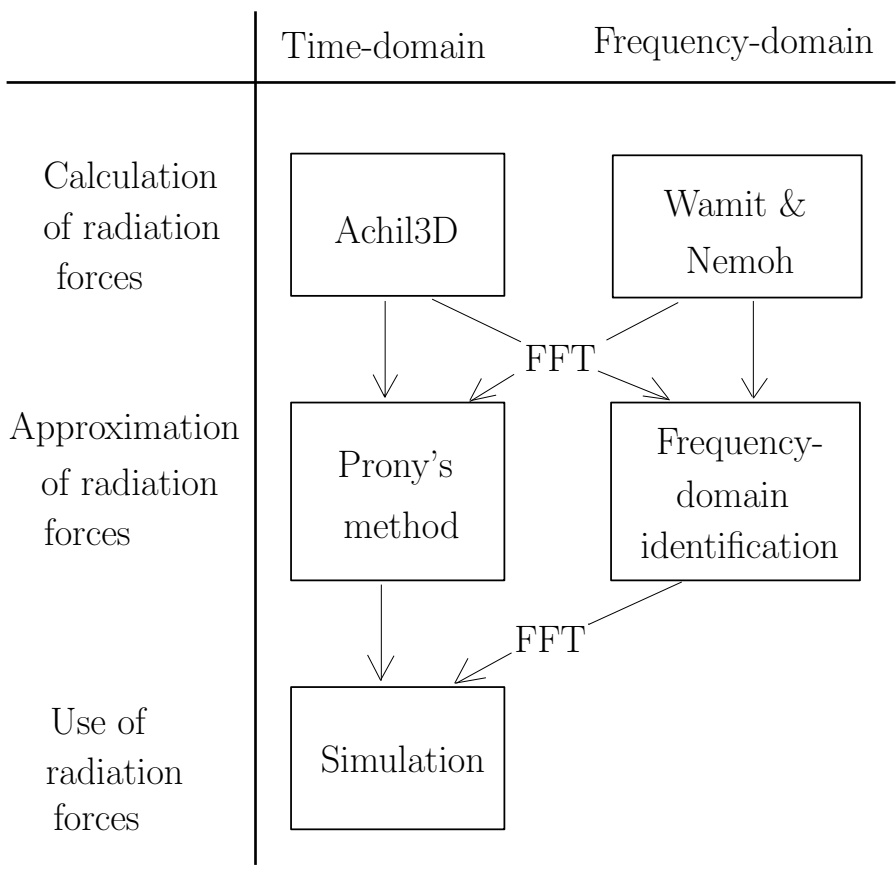

Figure 1: General overview of applicable steps to obtain radiation forces for time-domain simulations of wave energy converters and other floating marine structures.

The frequency-domain equivalent of equation (1) (Ogilvie 1964) is

$\boldsymbol{F}_{\text {exc }}(i \omega)=\left\{-\omega^{2}[\boldsymbol{M}+\boldsymbol{A}(\omega)]+i \omega \boldsymbol{B}(\omega)+\boldsymbol{C}\right\} \boldsymbol{X}(i \omega)$

In here matrices $\boldsymbol{A}(\omega)$ and $\boldsymbol{B}(\omega)$ contain the frequency dependent added mass- and radiation damping coefficients, respectively.

The relationships between added mass and radiation damping in the frequency-domain, and the impulse-response function in the time-domain, are known as Ogilvie's relationships (Ogilvie 1964, Taghipour et al. 2008):

$$
\begin{aligned}
& \boldsymbol{A}(\omega)=\boldsymbol{A}_{\infty}-\frac{1}{\omega} \int_{0}^{\infty} \boldsymbol{K}(t) \sin (\omega \tau) d \tau \\
& \boldsymbol{B}(\omega)=\int_{0}^{\infty} \boldsymbol{K}(t) \cos (\omega \tau) d \omega \\
& \boldsymbol{K}(t)=\frac{2}{\pi} \int_{0}^{\infty} \boldsymbol{B}(\omega) \cos (\omega t) d \omega
\end{aligned}
$$

\section{BOUNDARY ELEMENT METHODS}

\subsection{Frequency domain: Wamit, Nemoh}

Wamit and Nemoh are boundary element programs. Both programs are based on linear potential theory, which assumes the fluid to be homogeneous, incompressible and inviscid (Newman 1977, Faltinsen 1990). The flow in the fluid domain is assumed to be irrotational, and is described by the velocity potential $\phi$, which fulfills the Laplace equation (Lee 1995). The three-dimensional boundary value problem is solved by subdividing the submerged body surface into panels (WAMIT, Inc. 2013).

Wamit is a commercial software, which is used routinely to investigate wave effects on offshore structures and other marine vessels (Newman 2002). On the other hand, Nemoh is an open source software provided by Ecole Centrale de Nantes. Version 2.0 has been released in 2014 (Babarit 2014).

Wamit and Nemoh solve the linear potential flow problem in the frequency-domain. Both programs calculate the frequency dependent added mass and radiation damping coefficients in all six degrees of freedom, allowing the user to solve the radiation problem in the frequency-domain. The total hydrodynamic radiation force (Yu 1995, Taghipour et al. 2008) can be calculated by

$$
\boldsymbol{F}_{\text {rad }}(i \omega)=-[\boldsymbol{B}(\omega)+i \omega \boldsymbol{A}(\omega)] \dot{\boldsymbol{X}}(i \omega)
$$

One of the differences between Wamit and Nemoh is that Wamit is able to calculate the added mass for infinite and zero frequency. To the authors knowledge, the current Nemoh version does not have this feature.

\subsection{Time domain: Achil3D}

Achil3D is another boundary element software developed at Ecole Centrale de Nantes (Babarit 2010). As Wamit and Nemoh, Achil3D is based on linear potential theory. However, the fundamental difference is that Achil3D solves the boundary value problem in the time-domain rather than the frequency-domain.

Achil3D takes advantage of the fact that for linear time-domain hydrodynamics, the Green function satisfies a fourth order ordinary differential equation (Clement).

Achil3D calculates the impulse response function as well as the added mass for infinite frequency, allowing the user to evaluate the radiation problem directly in the time-domain. The total hydrodynamic radiation force (Falnes 2002) can be calculated by

$\boldsymbol{f}_{\text {rad }}(t)=-\boldsymbol{A}_{\infty} \ddot{\boldsymbol{x}}(t)-\int_{0}^{t} \boldsymbol{K}(t-\tau) \dot{\boldsymbol{x}}(\tau) d \tau$

\section{FINITE ORDER APPROXIMATIONS}

\subsection{Frequency domain: FD identification}

For the frequency-domain identification of the radiation problem of floating bodies, an open-source Matlab toolbox is available at www.marinecontrol.org (Perez 2009b, Fossen 2011). The toolbox is an independent part of the Marine Systems Simulator.

The frequency-domain representation of the retardation functions $\boldsymbol{K}(t)$ (Perez 2009a) in each degree of freedom is

$K_{i j}\left(i \omega_{k}\right)=B_{i j}\left(\omega_{k}\right)+i \omega_{k}\left[A_{i j}\left(\omega_{k}\right)-A_{\infty, i j}\right]$ 
In here, $K_{i j}$ can be approximated by a rational transfer function (Taghipour et al. 2008, Perez 2009b)

$\hat{K}_{i j}\left(i \omega_{k}, \boldsymbol{\theta}\right)=\frac{P_{i j}(s, \boldsymbol{\theta})}{Q_{i j}(s, \boldsymbol{\theta})}=\frac{p_{m} s^{m}+p_{m-1} s^{m-1}+\ldots+p_{0}}{s^{n}+q_{n-1} s^{n-1}+\ldots+q_{0}}$

were $\boldsymbol{\theta}$ is the vector of parameters

$\boldsymbol{\theta}=\left(p_{m}, \ldots, p_{0}, q_{n-1}, \ldots, q_{0}\right)^{T}$

Estimating the parameters in vector $\boldsymbol{\theta}$ can be posed as a complex least-squares curve fitting problem (Perez 2009a)

$\boldsymbol{\theta}^{*}=\underset{\boldsymbol{\theta}}{\arg \min } \sum_{k} w_{k} \epsilon_{k}^{*} \epsilon_{k}$

$\epsilon_{k}=K_{i j}\left(i \omega_{k}\right)-\hat{K}_{i j}\left(i \omega_{k}, \boldsymbol{\theta}\right)$

were the asterisk * indicates a transpose complex conjugate, and $w_{k}$ in equation (11) are weighting coefficients.

Once the approximation $\hat{K}_{i j}$ is found, the added mass and radiation damping coefficients (Perez 2009b) can be reconstructed

$\hat{A}_{i j}(\omega)=\operatorname{Im}\left\{\frac{1}{\omega} \hat{K}_{i j}(i \omega)\right\}+A_{\infty, i j}$
$\hat{B}_{i j}(\omega)=\operatorname{Re}\left\{\hat{K}_{i j}(i \omega)\right\}$

\subsection{Time domain: Prony's method}

Prony's method was developed by Baron de Prony in 1795, when he studied the expansion of different gases. The Prony analysis can be used to approximate the impulse response function in each degree of freedom with a sum of damped complex exponentials (Singh 2003)

$K_{i j}\left(t_{n}\right) \approx \hat{K}_{i j}(n)=\sum_{h=1}^{L} C_{h} \mu_{h}^{n}$

where $\mu_{h}=e_{h}^{a}$, and $n=0,1,2, \ldots, N-1$ is the number of equally spaced samples of the impulse response function. $L$ indicates the order of approximation. The coefficients $C_{h}$ and $\mu_{h}$ are calculated in three basic steps (Hildebrand 1956, Singh 2003): In the first step the linear prediction model

$d=D a$

is solved. In here, vector $\boldsymbol{d}$ and matrix $\boldsymbol{D}$ are build from the impulse response function to be approximated:

$\boldsymbol{d}=\left(\begin{array}{c}K_{i j}(L) \\ K_{i j}(L+1) \\ \vdots \\ K_{i j}(N-1)\end{array}\right)$

$$
\boldsymbol{D}=\left(\begin{array}{cclc}
K_{i j}(L-1) & K_{i j}(L-2) & \cdots & K_{i j}(0) \\
K_{i j}(L) & K_{i j}(L-1) & \cdots & K_{i j}(1) \\
\vdots & \vdots & \ddots & \vdots \\
K_{i j}(N-2) & K_{i j}(N-3) & \cdots & K_{i j}(N-L-1)
\end{array}\right)
$$

In general, the system in equation (16) is overdetermined, and can be solved approximately for $\boldsymbol{a}$ by least-squares methods. The components of vector $\boldsymbol{a}$

$\boldsymbol{a}=\left(\begin{array}{c}a_{1} \\ a_{2} \\ \vdots \\ a_{L}\end{array}\right)$

are the coefficients of the characteristic polynomial

$\mu^{L}-a_{1} \mu^{L-1}-a_{2} \mu^{L-2}-\ldots-a_{L-1} \mu-a_{L}=0$

The calculation of the roots $\mu_{h}$ of the characteristic polynomial (20) is the second basic step in Prony's analysis. The roots $\mu_{h}$ are subsequently used to build a system of linear equations

$$
\begin{gathered}
\boldsymbol{k}=\boldsymbol{M c} \\
\left(\begin{array}{c}
K_{i j}(0) \\
K_{i j}(1) \\
\vdots \\
K_{i j}(N-1)
\end{array}\right)=\left(\begin{array}{cccc}
\mu_{1}^{0} & \mu_{2}^{0} & \cdots & \mu_{L}^{0} \\
\mu_{1}^{1} & \mu_{2}^{1} & \cdots & \mu_{L}^{1} \\
\vdots & \vdots & \ddots & \vdots \\
\mu_{1}^{N-1} & \mu_{2}^{N-1} & \cdots & \mu_{L}^{N-1}
\end{array}\right)\left(\begin{array}{c}
C_{1} \\
C_{2} \\
\vdots \\
C_{L}
\end{array}\right)
\end{gathered}
$$

Solving this system for vector $c$ is the final basic step in Prony's analysis. Since the system (21) is overdetermined, again least-squares methods can be applied.

\section{APPLICATION EXAMPLE}

\subsection{Problem description}

The two methods to approximate the radiation problem in the frequency- and in the time-domain, which are described in section 3 are subsequently applied to a vertical cylinder in heave.

The cylinder has a draft $T=10 \mathrm{~m}$, and a radius $R=5 \mathrm{~m}$. The water depth $h$ is infinite and the density of the water is $\rho=1000 \frac{\mathrm{kg}}{\mathrm{m}^{3}}$. The origin of the coordinate system is located on the vertical axis of symmetry of the cylinder, at the mean free surface. As depicted in figure 2, the $\mathrm{z}$-axis of the coordinate system is pointing upwards. 


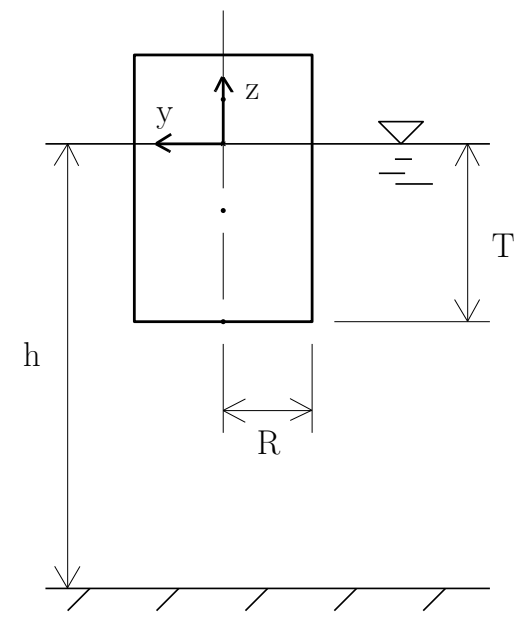

Figure 2: Vertical cylinder with a draft $T=10 \mathrm{~m}$ and a radius $R=5 \mathrm{~m}$. The water depth $h$ is infinite and the water density is $\rho=1000 \frac{\mathrm{kg}}{\mathrm{m}^{3}}$.

\subsection{Comparison of results in the time-and frequency domain}

The hydrodynamic properties for the cylinder described in section 4.1 are calculated by Wamit, Nemoh and Achil3D. Figure 3, 4 and 5 compare the results obtained from the three boundary element programs.

Figure 3 shows the frequency dependent added mass in heave $A_{33}(\omega)$, calculated by Wamit and Nemoh. Equation (3) was used to transform the impulse-response function $K_{33}(t)$, calculated by Achil3D, from the time-domain into the frequencydomain.

Figure 4 shows the radiation damping in heave $B_{33}(\omega)$, calculated by Wamit and Nemoh, versus the radian frequency. To transfer the impulse-response function $K_{33}(t)$, calulated by Achil3D into the frequency-domain, equation (4) was used.

In figure 5, the impulse response function $K_{33}(t)$ calculated by Achil3D is plotted. The frequency dependent radiation damping $B_{33}(\omega)$, calculated by Wamit and Nemoh was transferred into the timedomain using equation (5).

Figures 3, 4 and 5 show a very good agreement between the three different boundary element programs, both in the frequency- and in the time-domain.

Figures 6, 7 and 8 compare the approximations to Wamit output data in the time- and frequency-domain for different orders of approximation.

Figure 6 shows the frequency dependent added mass $A_{33}(\omega)$, calculated by Wamit, and the approximations of order 3 and 4, obtained from the frequency-domain identification Matlab toolbox.

In figure 7 , the frequency dependent radiation damping $B_{33}(\omega)$ calculated by Wamit, and the 3rd and 4th order approximations obtained from the frequency-domain identification Matlab toolbox, are plotted.

Figure 8 shows the impulse response function $K_{33}(t)$ obtained from Wamit data, which was transferred into the time-domain using equation (5). Order

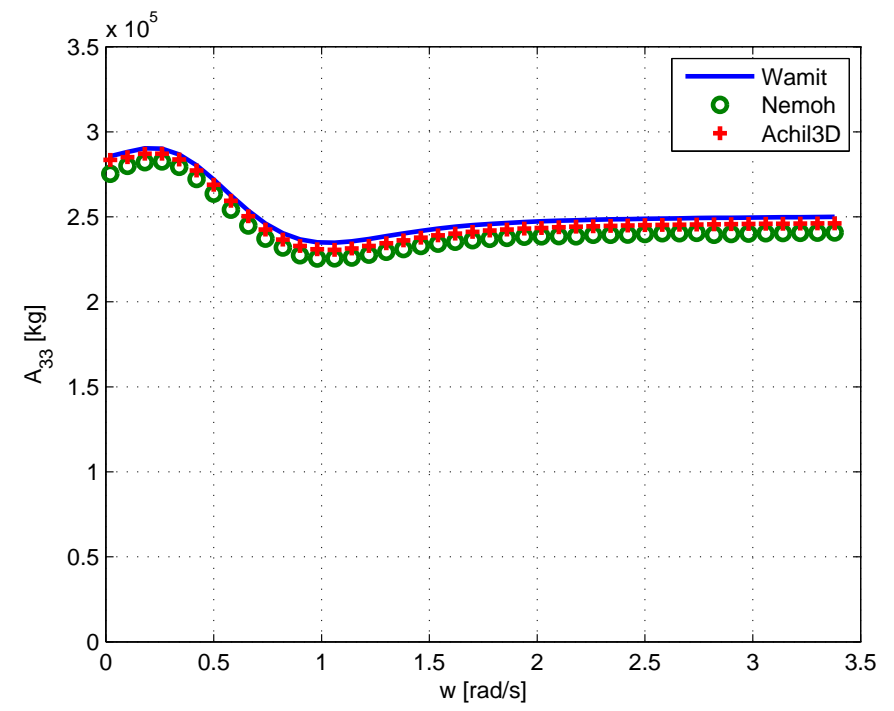

Figure 3: Added mass in heave $A_{33}(\omega)$, calculated by Wamit and Nemoh versus radian frequency. The Achil3D output is transferred into the frequency-domain using equation (3).

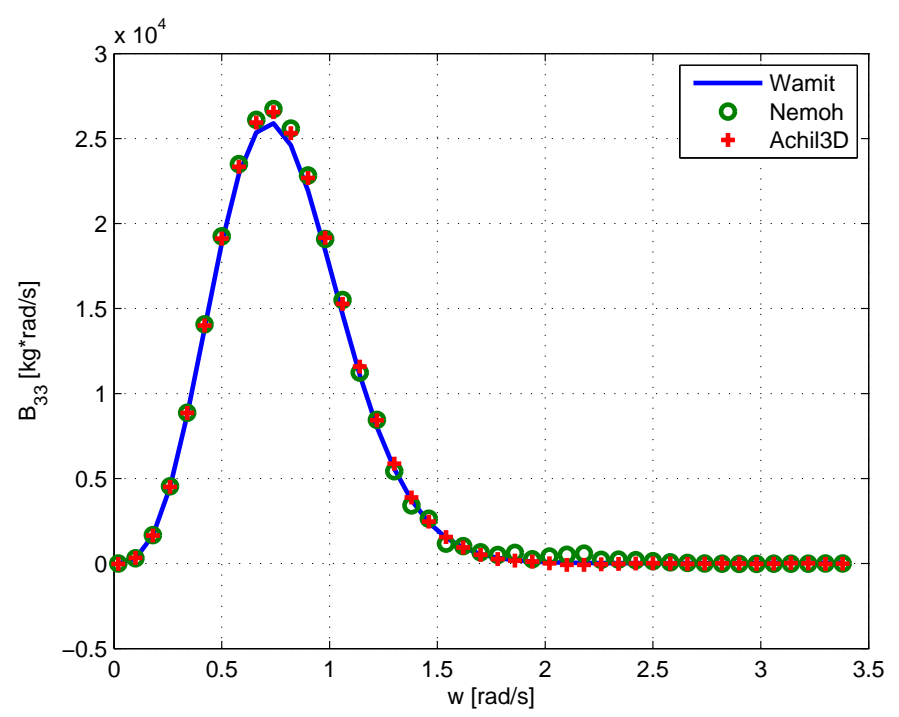

Figure 4: Radiation damping in heave $B_{33}(\omega)$, calculated by Wamit and Nemoh versus radian frequency. The output of Achil3D is transferred into the frequency-domain using equation (4).

3, 4 and 7 of the approximations to the impulse response function, using Prony's method, are also plotted in figure 8.

Figures 6,7 and 8 show a good agreement between the 4th order approximation and the approximated data, both in the frequency- and in the time-domain. A further increase of the approximation order increases the accuracy of the approximation, as depicted exemplary by the 7 th order approximation in figure 8 .

Figures 9,10 and 11 compare the results obtained from Achil3D data with their approximations in the time- and frequency-domain.

Figure 9 shows the added mass $A_{33}(\omega)$, obtained from Achil3D data, which was transferred from the time- into the frequency-domain using equation (3). The 3rd and 4th order approximations from the frequency-domain identification toolbox are also shown in figure 9. 


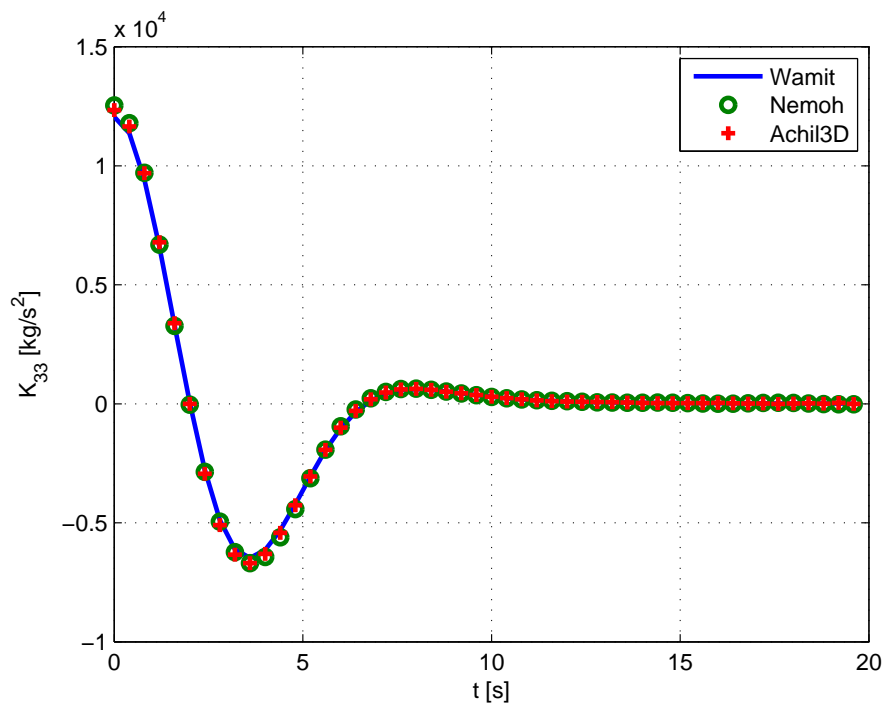

Figure 5: Impulse response function in heave $K_{33}(t)$, calculated by Achil3D. The output from Wamit and Nemoh is transferred into the time-domain using equation (5).

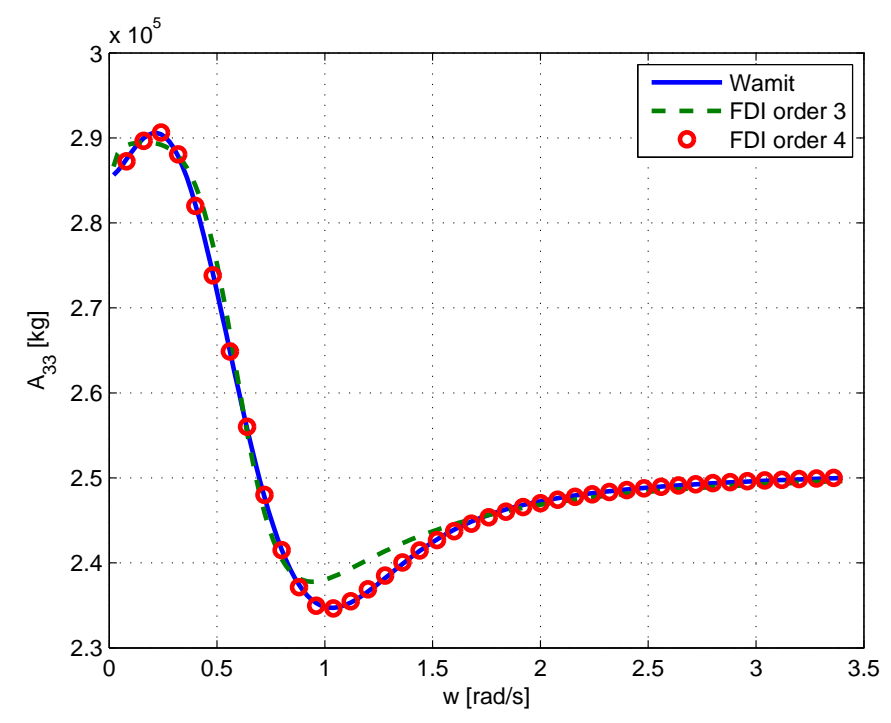

Figure 6: Added mass in heave $A_{33}(\omega)$, calculated by Wamit and the frequency-domain identification Matlab toolbox. The approximations of order 3 and 4 are plotted.

In figure 10 , the radiation damping $B_{33}(\omega)$ obtained from Achil3D data is plotted. The Achil3D output was transferred from the time- into the frequencydomain using equation (4). Figure 10 shows again the 3rd and 4th order approximations obtained by the frequency-domain identification Matlab toolbox.

Figure 11 shows the impulse response function $K_{33}(t)$ calculated by Achil3D, and the 3rd, 6th and 7th order of approximation using Prony's method.

Figures 9 and 10 show a good agreement between the 4th order approximation and the approximated data obtained from Achil3D. While the agreement between the 6th order approximation and the Achil3D data in figure 11 is good, the 7th order approximation shows a further increase of the accuracy of the approximation.

Figures 12 and 13 show the mean squared error (MSE) between the data obtained from Wamit and the approximations, versus the order of approximation.

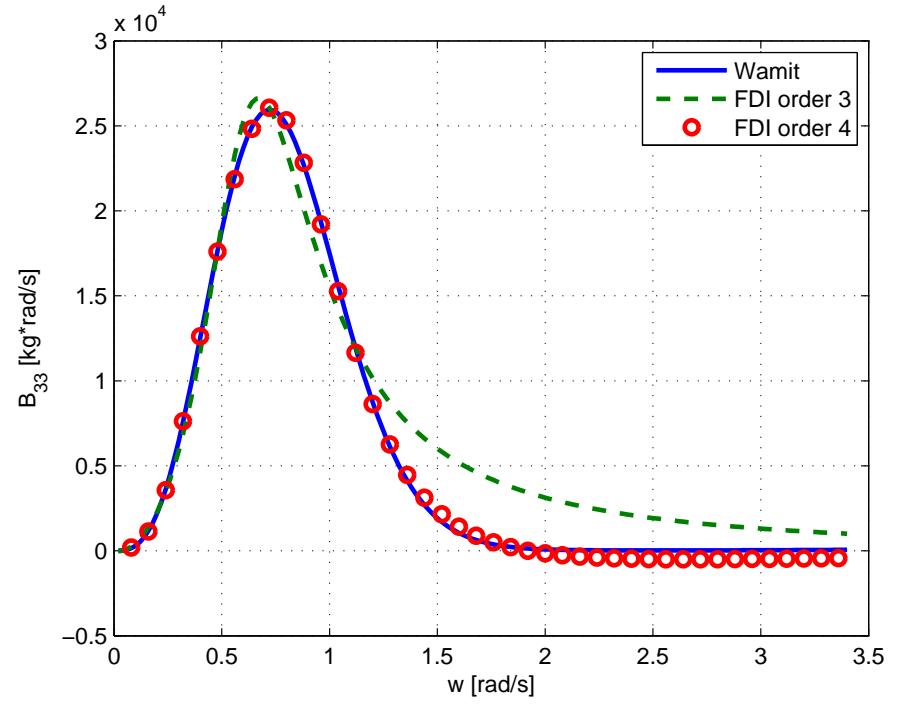

Figure 7: Radiation damping in heave $B_{33}(\omega)$, calculated by Wamit and the frequency-domain identification Matlab toolbox. The 3rd and 4th order approximations are shown.

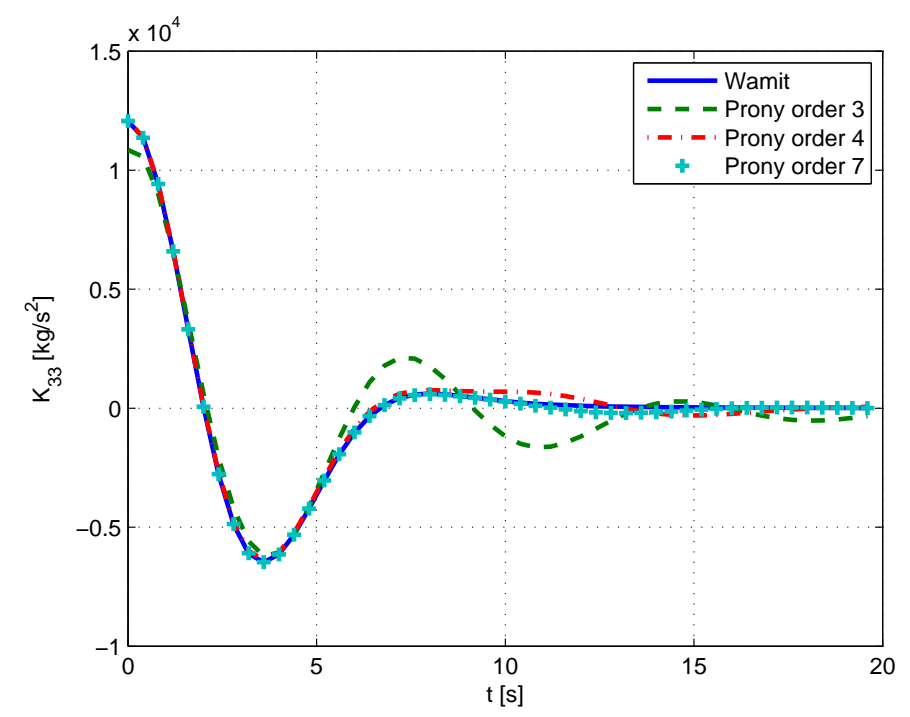

Figure 8: Impulse response function $K_{33}(t)$, calculated from Wamit output data and with Prony's method. The Prony approximations of order 3, 4 and 7 are plotted.

The MSE of the approximations to the added mass $A_{33}(\omega)$ and radiation damping $B_{33}(\omega)$ are shown in figure 12. While figure 13 shows the MSE of the approximations to the impulse response function $K_{33}(t)$. Both figure 12 and 13 show a significant difference between the MSE for order 3 and 4. This indicates that the accuracy gained by increasing the order of approximation from 4 to 5 is very small, compared to the gain in accuracy between order 3 and 4 . This observation is confirmed by figures 6,7 and 8 .

The MSE for the radiation damping $B_{33}(\omega)$ in figure 12 is larger for a third order approximation than it is for a 2 nd order approximation. The reason is that the frequency-domain identification toolbox approximates the added mass $A(\omega)$ and radiation damping $B(\omega)$ over a wider frequency range than the MSE is calculated for. The MSE is calculated over the frequency range for which hydrodynamic data is provided by the BEM. However, in addition to the lim- 


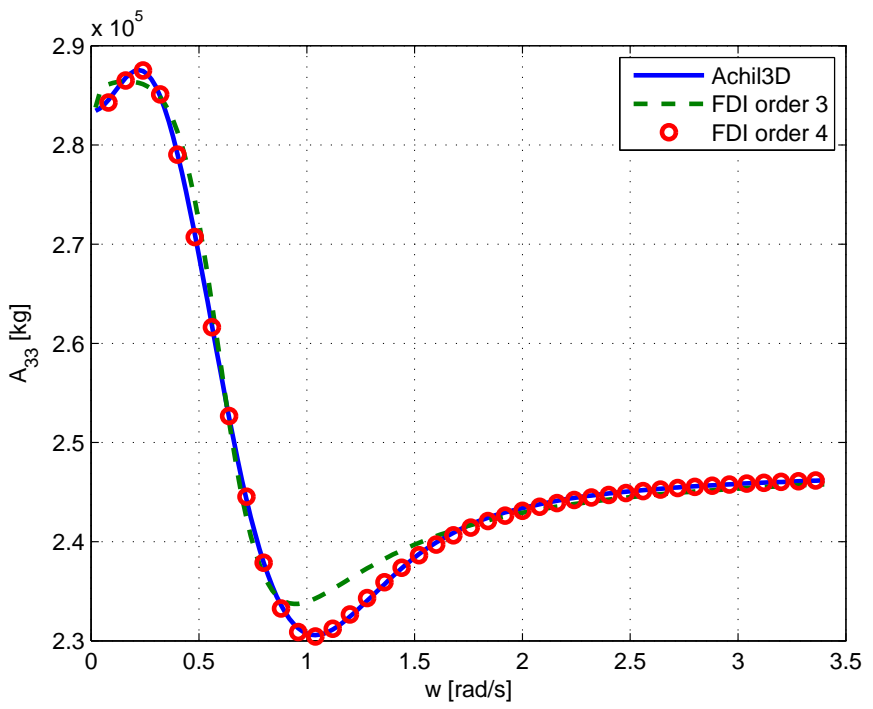

Figure 9: Added mass in heave $A_{33}(\omega)$, calculated from Achil3D data and the frequency-domain identification Matlab toolbox. The approximations of order 3 and 4 are plotted.

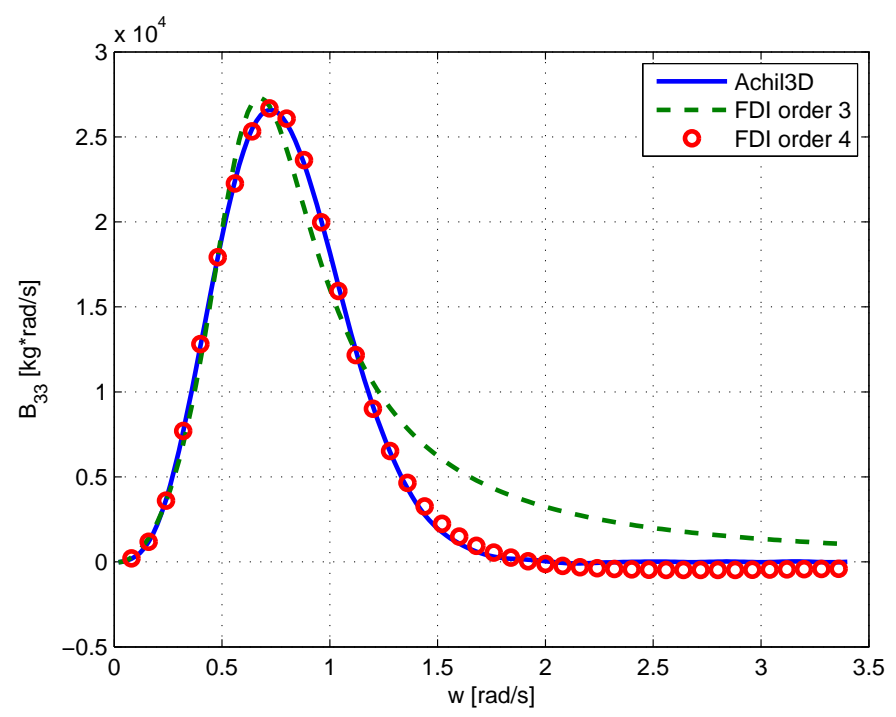

Figure 10: Radiation damping in heave $B_{33}(\omega)$, calulated from Achil3D output and the frequency-domain identification Matlab toolbox. The 3rd and 4th order of the approximation are shown.

ited frequency range for which hydrodynamic data is available, the frequency-domain identification toolbox interpolates, and subsequently, approximates data between this limited frequency range and the infinite frequency. This gives the impression that an increased model order results in a lower accuracy.

Figures 14 and 15 show the MSEs between the data obtained from Achil3D and the approximations, versus the order of approximation. While figure 14 shows the MSE of the approximations to the added mass $A_{33}(\omega)$ and radiation damping $B_{33}(\omega)$, figure 15 shows the MSE of the approximation to the impulse response function $K_{33}(t)$.

Similar to figure 12 , the MSE of the radiation damping $B_{33}(\omega)$ in figure 14 increases from a second order approximation to a third order approximation. As mentioned in the discussion of figure 12, the reason is again the limited frequency range over which the MSE is calculated.

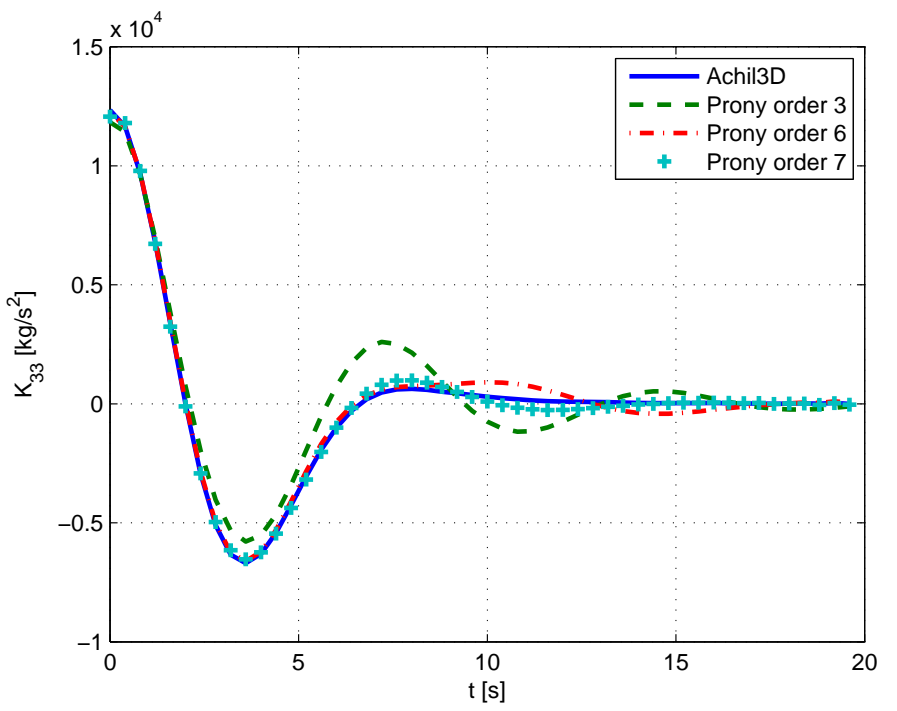

Figure 11: Impulse response function $K_{33}(t)$, calculated by Achil3D and with Prony's method. The Prony approximations of order 3, 6 and 7 are shown.

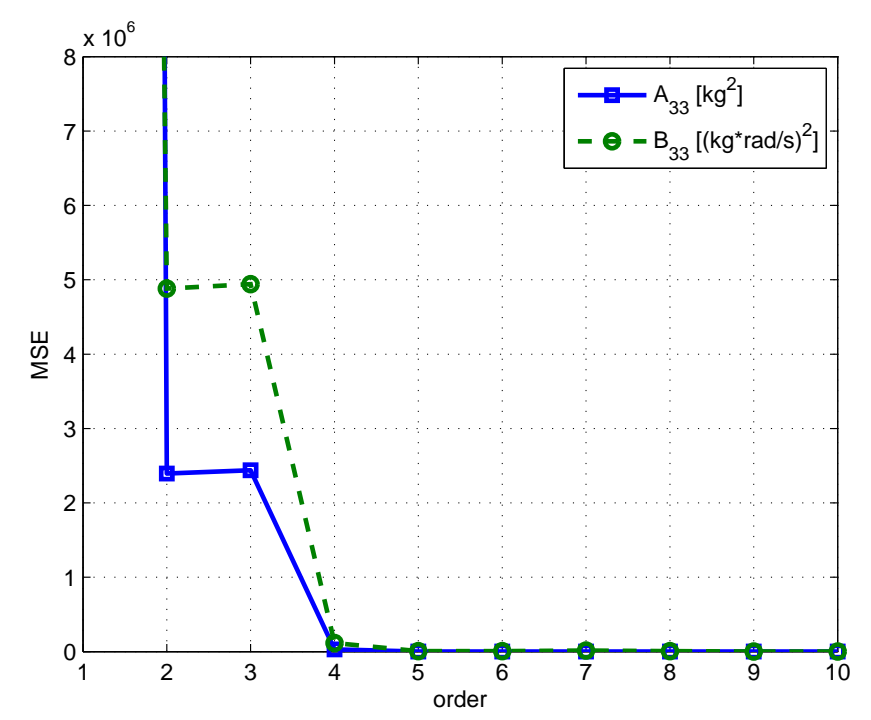

Figure 12: Mean squared error of the added mass $A_{33}(\omega)$ and radiation damping $B_{33}(\omega)$ between the Wamit data and the approximations from frequency-domain identification. The mean squared error is plotted versus the order of approximation.

While the results of Wamit and Achil3D show good agreement, applying Prony's method to hydrodynamic data, obtained from both BEMs, leads to different accuracies for the same approximation order, as depicted in figure 15 . In both cases very similar hydrodynamic data was approximated, as shown in figure 5. However, small differences in the hydrodynamic data can cause large differences in the approximation. As depicted in figure 13, the significant decrease of the MSE which occurs between order three and four in the case of Wamit data, occurs between order five and six in the case of Achil3D data, as shown in figure 15. Furthermore, figure 15 shows that the MSE between the Achil3D data and the approximation increases for order eight and nine. To further investigate the reasons for this increase of the MSE figures 16 and 17 are plotted. Both figures, but in particular figure 17 , show a significant decrease in the mag- 


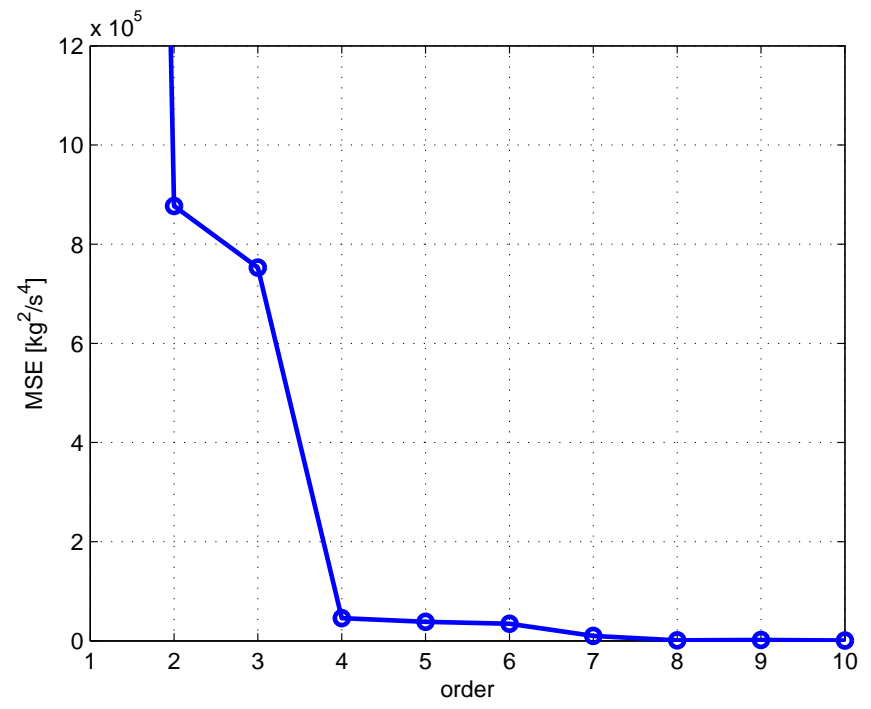

Figure 13: Mean squared error between the impulse response function $K_{33}(t)$ obtained from Wamit data and the approximations from Prony's method. The mean squared error is plotted versus the order of approximation.

nitude of the singular value from order two to order three. Beyond order three the singular values change very little. This suggests that increasing the order of approximation beyond order three causes the matrices $\boldsymbol{D}$ and $\boldsymbol{M}$ in Prony's method to become almost singular, which causes the approximation problem to be ill conditioned. This can be confirmed by the fact that matrices $\boldsymbol{D}$ and $\boldsymbol{M}$ have full rank for each order of approximation, with the only exceptions being matrix $M$ for order eight and nine.

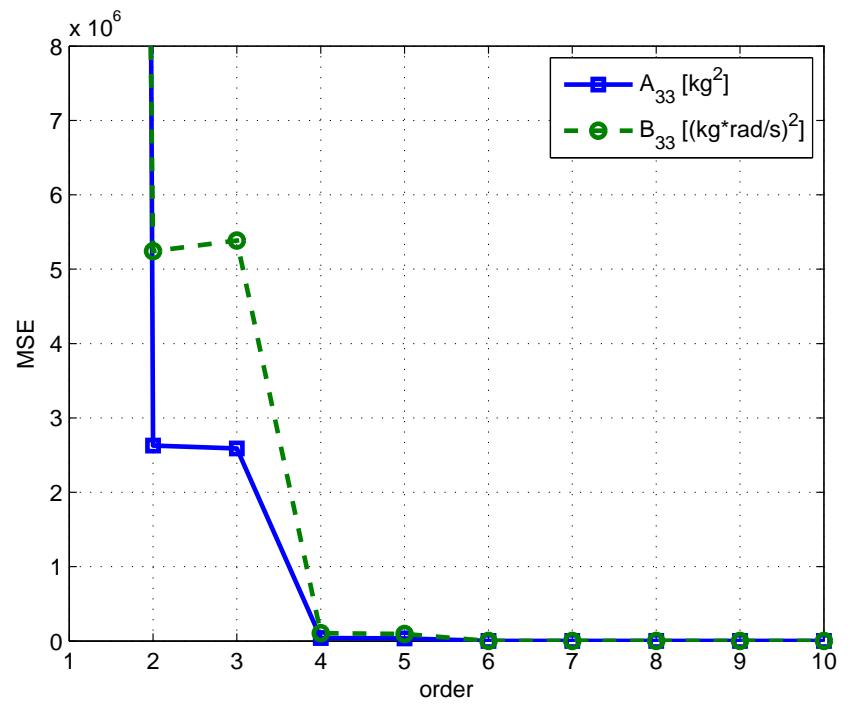

Figure 14: Mean squared error of the added mass $A_{33}(\omega)$ and radiation damping $B_{33}(\omega)$ calculated from Achil3D data and the approximations from frequency-domain identification. The mean squared error is plotted versus the order of approximation.

\subsection{Comparison of results with respect to the computational cost}

This section compares the computational costs of the different hydrodynamic software packages, and of the approximation methods used.

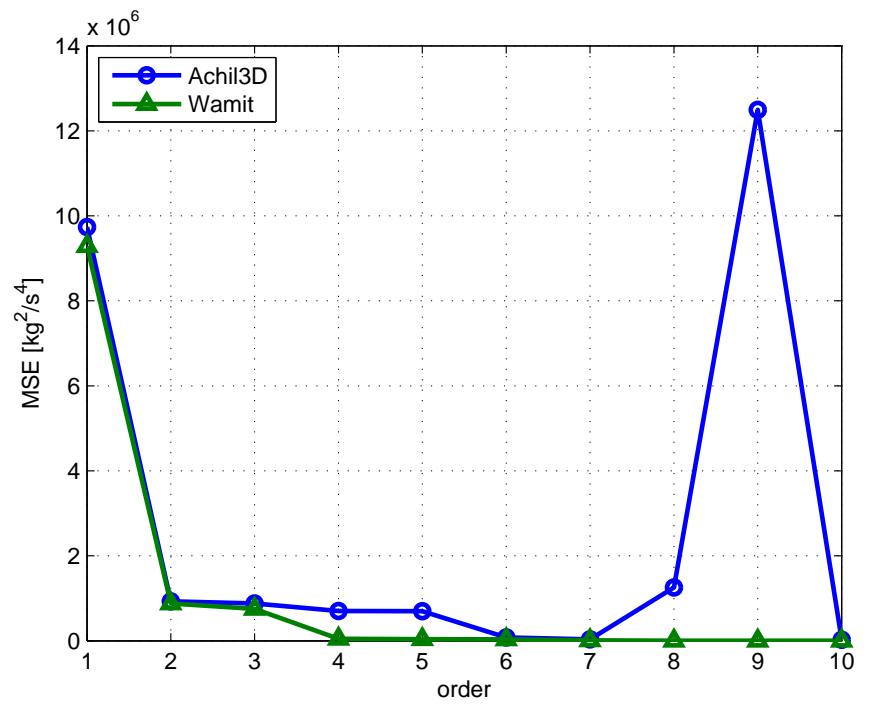

Figure 15: Mean squared error between the impulse response function $K_{33}(t)$ calculated by Achil3D and the approximations from Prony's method. The mean squared error is plotted versus the order of approximation. For easier comparison the MSE from figure 13 is also plotted again.
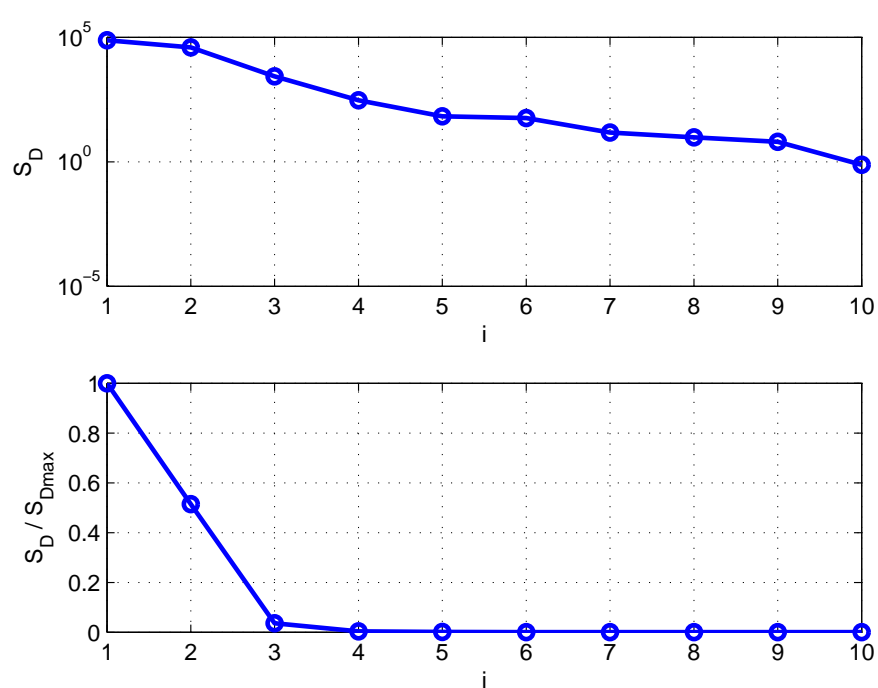

Figure 16: Singular values of matrix $\boldsymbol{D}$ in Prony's method using Achil3D data. The upper part shows the singular values on a logarithmic scale and the lower part shows the singular values normalized by the maximum singular value.

All computations were carried out on a Dell Optiplex 755 PC. The PC has a $2.53 \mathrm{GHz}$ dual core processor and 3GB RAM. For all computations the PC was limited to $50 \%$ of its capacity.

Table 1: Runtimes of the three boundary element software packages.

\begin{tabular}{lll}
\hline Software & \multicolumn{2}{c}{ Runtimes } \\
& heave only & $6 \mathrm{DOF}$ \\
\hline Wamit & $16 \mathrm{~s}$ & 42 \\
Nemoh & $574 \mathrm{~s}$ & $875 \mathrm{~s}$ \\
Achil3D & $/$ & $4774 \mathrm{~min}$ \\
\hline
\end{tabular}

Table 1 lists the run times required to calculate the hydrodynamic parameters with the three different BEMs. The geometry considered for the comparison of computational costs is again the vertical cylinder described in section 4.1 . The cylinder surface 

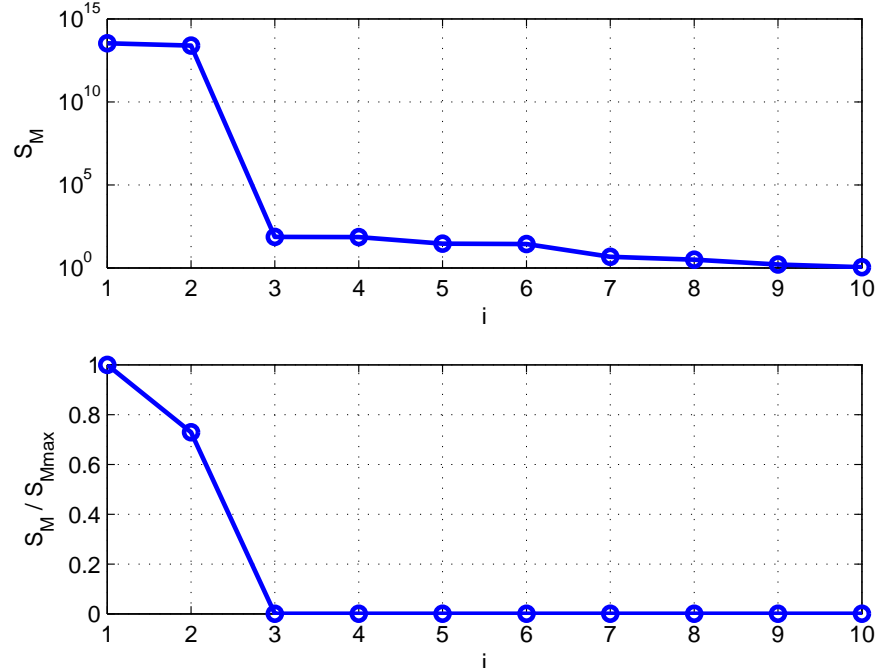

Figure 17: Singular values of matrix $M$ in Prony's method using Achil3D data. The upper part shows the singular values on a logarithmic scale and the lower part shows the singular values normalized by the maximum singular value.

was subdivided into 1000 panels. For the Wamit and Nemoh runs 300 radian frequencies, equally spaced between 0.0117 and $3.5 \mathrm{rad} / \mathrm{s}$, were calculated. For the Achil3D run the impulse response was calculated for $20 \mathrm{~s}$ with a time interval of $0.01 \mathrm{~s}$. Achil3D's run time of 4774 minutes, compared to 875 seconds for Nemoh and 42 seconds for Wamit, in the case of 6 degrees of freedom (DOF), indicates the significantly longer time required to solve the boundary value problem directly in the time-domain rather than the frequency-domain. Considering heave only, the run time of Wamit is $16 \mathrm{~s}$, whereas Nemoh takes $574 \mathrm{~s}$ to run. To the author's knowledge, Achil3D runs for $6 \mathrm{DOF}$ only.

The computation of the 7th order approximations, both in the time- and the frequency-domain, requires less than one second of CPU time.

\section{CONCLUSION}

This paper examined different routes through the radiation force modeling landscape depicted in figure 1, on an application example. For this purpose the results of three different boundary element programs were compared in the time- and in the frequencydomain. For a vertical cylinder in heave the three programs, namely Wamit, Nemoh and Achil3D, show very good agreement.

The three boundary element programs were also compared with respect to their computational cost, showing that the solution of the boundary value problem directly in the time-domain is much more demanding than the solution in the frequency-domain.

Compared to Wamit, Nemoh has the disadvantage of longer computational times required. However, Nemoh has the huge advantage that it is a freely available open source software.
Two different methods to approximate the fluidmemory effect, related to the radiation problem of floating bodies, were reviewed. For the approximation in the time-domain Prony's method was used. In the frequency-domain, the frequency-domain identification Matlab toolbox was used for the approximation. One purpose of this paper was to show the influence of the approximation order on the accuracy of the approximation, in the frequency- as well as the timedomain. From the discussion of figure 15 to 17 it can be concluded that increasing the approximation order does not necessarily increase the accuracy of the approximation.

Even though the results of frequency- and timedomain BEMs show good agreement, the use of these results in Prony's method can lead to different accuracies for the same approximation order, as shown in figure 15.

\section{REFERENCES}

Babarit, A. (2010). Achil3D v2.011 user manual. Ecole Centrale de Nantes.

Babarit, A. (2014). Nemoh user manual. Ecole Centrale de Nantes.

Clement, A. Using differential properties of the green function in seakeeping computational codes.

Cummins, W. (1962). The impulse response function and ship motions. Schiffstechnik 9, 101-109.

Falnes, J. (2002). Ocean waves and oscillating systems. Cambridge University Press.

Faltinsen, O. (1990). Sea loads on ships and offshore structures. Cambridge University Press.

Fossen, T. (2011). Handbook of marine craft hydrodynamics and motion control. John Wiley \& Sons, Ltd.

Hildebrand, F. (1956). Introduction to numerical analysis. Tata McGraw-Hill Publishing Company Ltd.

Lee, C. (1995). Wamit theory manual. Massachusetts Institute of Technology, Department of Ocean Engineering.

Newman, J.N. \& Lee, C. (2002). Boundary element methods in offshore structure analysis. Journal of Offshore Mechanics and Arctic Engineering 124, 81-89.

Newman, J. (1977). Marine hydrodynamics. MIT Press.

Ogilvie, T. (1964). Recent progress toward the understanding and prediction of ship motions. In Sixth Symposium on Naval Hydrodynamics.

Perez, T. \& Fossen, T. (2009a). Identification of dynamic models of marine structures from frequency-domain data enforcing model structure and parameter constraints. Technical report, ARC Centre of Excellence for Complex Dynamic Systems and Control.

Perez, T. \& Fossen, T. (2009b). A matlab toolbox for parametric identification of radiation-force models of ships and offshore structures. Modeling, Identification and Control 30, 1-15.

Singh, S. (2003). Application of prony analysis to characterize pulsed corona reactor measurements. Master's thesis, University of Wyoming.

Taghipour, R. et al. (2008). Hybrid frequency-time domain models for dynamic response analysis of marine structures. Ocean Engineering 35, 685-705.

WAMIT, Inc. (2013). WAMIT User Manual Version 7.0. WAMIT, Incorporated and Massachusetts Institute of Technology.

Yu, Z. \& Falnes, J. (1995). State-space modelling of a vertical cylinder in heave. Applied Ocean Research 17, 265-275. 